\title{
Clinical Outcomes of Minimally Invasive Surfactant Therapy via Tracheal Catheterization in Neonates with a Gestational Age of 30 Weeks or More Diagnosed with Respiratory Distress Syndrome
}

\author{
Moon Young Seo, Gyu Hong Shim, and Myoung Jae Chey \\ Department of Pediatrics, Inje University Sanggye Paik Hospital, Inje University College of Medicine, Seoul, Korea
}

\section{ABSTRACT}

Purpose: Minimally invasive surfactant therapy (MIST) is currently used as a method of surfactant replacement therapy (SRT) for the treatment of respiratory distress syndrome (RDS) in preterm infants with a gestational age of less than 30 weeks. However, few studies have been conducted on MIST in neonates with a gestational age of 30 weeks or more. In this study, we compared MIST with endotracheal intubation as a rescue SRT for spontaneously breathing neonates with a gestational age of 30 weeks or more who were diagnosed with RDS.

Methods: We investigated the clinical characteristics of spontaneously breathing neonates admitted to the neonatal intensive care unit of the Inje University Sanggye Paik Hospital from January 1, 2014 to December 31, 2016. These neonates were born at a gestational age of 30 weeks or more and were diagnosed with RDS. The neonates who were administered surfactant by MIST were categorized into the MIST group $(n=16)$ and those who underwent endotracheal intubation were categorized into the control group $(\mathrm{n}=45)$. Thereafter, the clinical characteristics between the groups were compared.

Results: Compared to the control group, the MIST group was less likely to require mechanical ventilation within 72 hours $(P<0.001)$. The frequency of bradycardia during SRT was also low in the MIST group $(P=0.033)$.

Conclusion: MIST is considered relatively feasible and safe for treating RDS for reducing the need for mechanical ventilation and decreasing the occurrence of bradycardia during surfactant administration in neonates with a gestational age of 30 weeks or more.

Key Words: Noninvasive ventilation, Minimally invasive surfactant therapy, Respiratory distress syndrome, Mechanical ventilation, Bradycardia

\section{INTRODUCTION}

Respiratory distress syndrome (RDS) is one of the most common neonatal respiratory
Received: 17 May 2018

Revised: 15 August 2018

Accepted: 16 August 2018

Correspondence to: Gyu Hong Shim Department of Pediatrics, Inje University Sanggye Paik Hospital, Inje University College of Medicine, 1342 Dongil-ro, Nowon-gu, Seoul 01757, Korea

Tel: +82-2-950-1632

Fax: +82-2-950-1246

E-mail: peddoc@paik.ac.kr

Copyright(c)

By Korean Society of Neonatology.

All right reserved.

This is an Open-Access article distributed under the terms of the Creative Commons Attribution Non-Commercial License (http://creativecommons.org/licenses/ by-nc/4.0), which permits unrestricted non-commercial use, distribution, and reproduction in any medium, provided the original work is properly cited. 
diseases, and it is an important cause of neonatal respiratory morbidity and mortality ${ }^{1)}$. The treatment of neonatal RDS requires invasive or noninvasive ventilation with surfactant replacement therapy (SRT). It is now recognized that primary nasal continuous positive airway pressure (CPAP) is a reasonable alternative to early intubation and/or SRT in preterm infants with respiratory distress after birth $^{2,3)}$. Increased use of CPAP as a primary therapy for neonates with respiratory distress is accompanied by the dilemma regarding the exact time and method of starting SRT. To overcome this problem, Verder et al. ${ }^{4,5)}$ devised the INtubation-SURfactantExtubation (INSURE) method, which has been reported to reduce the frequency and duration of invasive ventilation. However, the INSURE method is associated with problems such as the need for endotracheal intubation, positive pressure ventilation (PPV), and sedation $^{6-9)}$. Thus, many neonatologists are investigating minimally invasive surfactant therapy (MIST) to overcome these problems $^{10)}$.

To date, there have been many studies on preterm infants with a gestational age of 24 to 29 weeks who were diagnosed with RDS and were administered surfactant by MIST ${ }^{10)}$. However, few studies have reported on moderately preterm infants, late preterm infants, and term infants in whom RDS was treated via MIST. Accordingly, we aimed to compare the clinical outcomes of the MIST group (SRT by MIST) and control group (SRT by endotracheal intubation) to determine the optimal rescue SRT in the management of RDS for spontaneously breathing neonates with a gestational age of 30 weeks or more.

\section{MATERIALS AND METHODS}

This was a single-center, retrospective study conducted in the neonatal intensive care unit (NICU) of the Inje University Sanggye Paik Hospital from January 1, 2014 to December 31, 2016. The study protocol was reviewed and approved by the Institutional Review Board (IRB) of the hospital (approval number: SGPAIK 2017-08-008). The IRB waived the need for informed consent for this retrospective chart review.

\section{Study population}

Infants who were admitted to the NICU of Inje University Sanggye Paik Hospital at a gestational age of 30 weeks and over and who required respiratory support within 2 hours after birth were screened from January 1, 2014 to December 31, 2016. The study period was set since January 1, 2014, when MIST was introduced in this hospital. The MIST method includes tracheal catheterization, intrapharyngeal instillation, and aerosolization. Among the various MIST methods, our clinic uses the Cologne method $^{10)}$. This method uses a 4 to 5 FG (French Gauge) gastric tube as a conduit for insertion into the airway. The procedure involves checking the vocal cords using laryngoscopy, insertion of the gastric tube into the airway using Magill forceps, and administration of the surfactant ${ }^{10)}$. The following infants were included in the study: (1) spontaneously breathing infants who were born at no less than 30 weeks of gestation and (2) infants diagnosed with $\mathrm{RDS}$, who required a fraction of inspired oxygen of $\geq 0.35$ and who were administered pulmonary surfactant within 48 hours. Infants who were transferred to other hospitals, those who required aggressive resuscitation such as endotracheal intubation in the delivery room, and those diagnosed with chro mosomal abnormalities, or multiple congenital anomalies were excluded.

Infants who had received a pulmonary surfactant (200 mg/ kg Curosurf ${ }^{\circledR}$, Chiesi Pharmaceuticals, Parma, Italy) for the treatment of RDS were divided into two groups: the MIST group and the control group. The MIST group was defined as the group in which the surfactant was administered by MIST via a sterile 5-French, orogastric tube while receiving CPAP (peek end-expiratory pressure, 5 to $8 \mathrm{~cm} \mathrm{H}_{2} \mathrm{O}$ ) without endotracheal intubation. This protocol was based on the MIST procedure described by Gopel et al. ${ }^{11)}$. The control group was defined as the group in which the surfactant was administered by the conventional endotracheal intubation procedure. Subsequently, each group was subdivided into two groups according to gesta tional age, with neonates with gestational age less than 35 weeks in one subgroup and those with age $\geq 35$ weeks in the other group.

Prenatal parameters such as twin delivery, type of delivery, birth in our clinic, nulliparity, maternal age, maternal diabetes mellitus, maternal hypertension, use of antenatal steroids, premature rupture of membrane (PROM), and pathologic chorioamnionitis were reviewed. In addition, neonatal clinical characteristics such as gestational age, birth weight, small for gestational age (SGA, defined as birth weight below the 10th percentile for infants of the same gestational age), large for gestational age (LGA, defined as birth weight above the 90th percentile for infants of the same gestational age), sex, Apgar score (1- and 5-minute), meconium staining, and need for PPV support 
at birth were studied. Treatment data including time interval from birth to surfactant administration, failure to administer procedure on the first attempt, need for mechanical ventilation within 72 hours of birth or at any time, need for multiple doses of surfactant, bradycardia during surfactant administration, need for PPV during surfactant administration, duration of respiratory support, and duration of hospital stay were collected. Neonatal morbidities such as air-leak syndrome, pulmonary hemorrhage, patent ductus arteriosus (PDA), necrotizing enterocolitis (NEC), retinopathy of prematurity (ROP), intraventricular hemorrhage (IVH), hypoxic brain injury, bronchopulmonary dysplasia (BPD), use of antibiotic therapy for suspected infection, and culture pro ven sepsis were reviewed. Thereafter, we compared the results between groups through statistical analysis.

\section{Primary and secondary outcomes}

The primary outcomes of this study were need for mechanical ventilation within 72 hours of birth or at any time (failure of non-invasive respiratory support) and requirement of additional surfactant. Additional surfactant supplementation was considered in patients without clinical improvement even after SRT, consistent between the groups. The secondary out comes were bradycardia during surfactant administration, air-leak syndrome, pulmonary hemorrhage, BPD, duration of mechanical ventilation, duration of CPAP, and duration of heated humidified high-flow nasal cannula. In addition, hospital days, PDA (requiring treatment), NEC (higher than stage 2), ROP, IVH (higher than stage 2), hypoxic brain injury, use of antibiotic therapy for suspected infection, and culture proven sepsis were investigated.

\section{Definitions}

The diagnosis of RDS was confirmed by a pediatric radiologist after observing reduced lung volumes, a reticulogranular pattern of lung consolidation, and air bronchograms ${ }^{12}$. Pulmonary hemorrhage was diagnosed on obtaining blood-tinge tracheal aspirate and the need for increasing fraction of inspired oxygen. BPD was defined according to the definition of the National Institutes of Health Consensus ${ }^{13)}$. NEC was diagnosed as stage 2 or more, according to modified Bell's staging ${ }^{14)}$. International Classification of Retinopathy of Prematurity was used to classify $\mathrm{ROP}^{15)}$. Grading of IVH was carried out as per the Papile grading system $^{16)}$.

\section{Statistical analysis}

Continuous variables were analyzed using Student's $t$-test or Mann-Whitney $U$-test. Chi-square test or Fisher's exact test was used for categorical variables. In addition, multiple regression analysis was performed on related factors with a $P$-value below 0.05 in the univariate analysis. All analyses were considered statistically significant when the $P$-value was less than 0.05 . Statistical analysis was carried out using the SPSS software version 19.0 for Windows (IBM Co., Armonk, NY, USA).

\section{RESULTS}

We investigated the perinatal characteristics and neonatal outcomes of 307 neonates who were born at no less than 30 weeks of gestation and had respiratory distress within 2 hours of birth from January 1, 2014 to December 31, 2016. Infants who died during hospitalization $(\mathrm{n}=3)$, infants who were transferred to other hospitals $(n=2)$, neonates who required aggressive resuscitation such as tracheal intubation in the delivery room $(\mathrm{n}=2)$, and neonates already diagnosed with persistent pulmo nary hypertension of the newborn or meconium aspiration syndrome at birth $(\mathrm{n}=3)$ were excluded from the study. Of the 297 newborns, those who were administered surfactant for the treatment of RDS ( $n=61)$ were divided into two groups: the gestational age group of 30 to 35 weeks $(n=26)$ and the gestational age group of 35 weeks or more $(n=35)$. Thereafter, each group was further divided into two groups, the MIST group and the control group (Figure 1).

\section{Perinatal characteristics}

Baseline demographic data analysis showed no significant difference between the MIST and control groups with respect to gestational age, birth weight, twin delivery, C-section, nulliparity, maternal age, maternal diabetes, maternal hypertension, ante natal steroid use, sex, LGA, SGA, 1- and 5-minute Apgar scores, PROM, pathologic chorioamnionitis, need for PPV at birth, and time interval from birth to surfactant administration. Of the total study population, the neonates who were born at our hospital were significantly more likely to receive MIST $(P=0.036)$ (Table 1).

\section{Neonatal outcomes}

The MIST group was significantly less likely to receive mechanical ventilation within the hospitalization period than the 


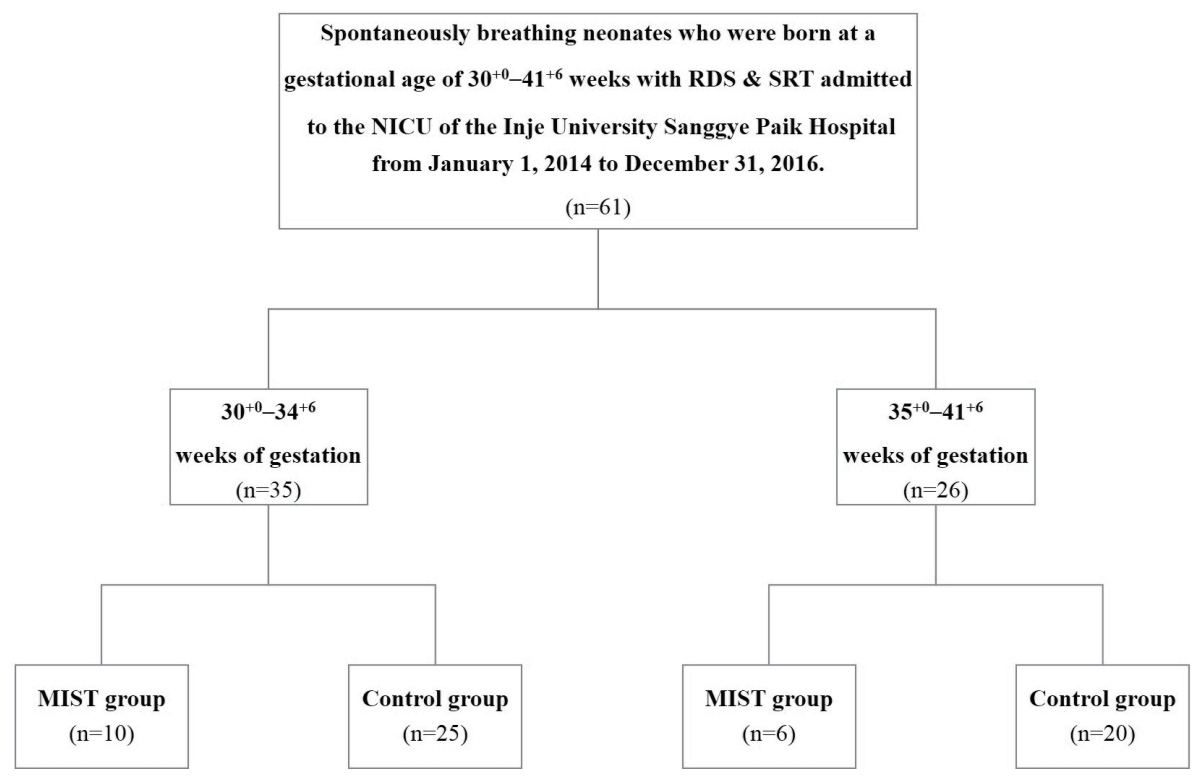

Figure 1. Study population of this study and subgrouping by minimally invasive surfactant therapy (MIST) and control groups. Abbreviations: RDS, respiratory distress syndrome; SRT, surfactant replacement therapy; NICU, neonatal intensive care unit.

Table 1. Baseline Demographic Data of Each Group

\begin{tabular}{|c|c|c|c|c|c|c|c|c|c|}
\hline \multirow[b]{2}{*}{ Baseline demographic } & \multicolumn{3}{|c|}{ Total $(n=61)$} & \multicolumn{3}{|c|}{ GA $30^{+0}-34^{+6}(n=35)$} & \multicolumn{3}{|c|}{ GA $35^{+0}-41^{+6}(n=26)$} \\
\hline & $\begin{array}{c}\text { MIST } \\
(n=16)\end{array}$ & $\begin{array}{l}\text { Control } \\
(n=45)\end{array}$ & $\begin{array}{c}P \text { - } \\
\text { value }\end{array}$ & $\begin{array}{c}\text { MIST } \\
(\mathrm{n}=10)\end{array}$ & $\begin{array}{c}\text { Control } \\
(n=25)\end{array}$ & $\begin{array}{c}P \text { - } \\
\text { value }\end{array}$ & $\begin{array}{l}\text { MIST } \\
(\mathrm{n}=6)\end{array}$ & $\begin{array}{c}\text { Control } \\
(\mathrm{n}=20)\end{array}$ & $\begin{array}{c}P \text { - } \\
\text { value }\end{array}$ \\
\hline Gestational age (wk) & $33.9 \pm 2.6$ & $34.6 \pm 2.8$ & 0.382 & $32.3 \pm 1.7$ & $32.4 \pm 1.3$ & 0.850 & $36.5 \pm 1.6$ & $37.3 \pm 1.3$ & 0.223 \\
\hline Birth weight (g) & $2,272.2 \pm 757.6$ & $2,331.7 \pm 736.7$ & 0.784 & $1,885.0 \pm 511.7$ & $1,777.0 \pm 368.5$ & 0.489 & $2,917.5 \pm 671.6$ & $3,025.0 \pm 417.8$ & 0.636 \\
\hline Twin delivery & 0 & $8(18)$ & 0.097 & 0 & $7(28)$ & 0.084 & 0 & $1(5)$ & 1.000 \\
\hline Cesarean section & $13(81)$ & $36(80)$ & 1.000 & $9(90)$ & $18(72)$ & 0.390 & $4(67)$ & $18(90)$ & 0.218 \\
\hline Inborn & $14(88)$ & $26(58)$ & 0.036 & $10(100)$ & $22(23)$ & 0.542 & $4(67)$ & $4(20)$ & 0.051 \\
\hline Nulliparous & $10(63)$ & $20(44)$ & 0.902 & $6(60)$ & $12(48)$ & 0.711 & $4(67)$ & $8(40)$ & 0.365 \\
\hline Maternal age (yr) & $33.5 \pm 3.7$ & $33.0 \pm 3.7$ & 0.656 & $33.1 \pm 4.1$ & $31.7 \pm 3.6$ & 0.331 & $34.2 \pm 3.3$ & $34.7 \pm 3.1$ & 0.741 \\
\hline Maternal DM & $5(31)$ & $6(13)$ & 0.109 & $3(30)$ & $4(16)$ & 0.381 & $2(33)$ & $2(10)$ & 0.218 \\
\hline Maternal HTN & $3(19)$ & $2(4)$ & 0.108 & $3(30)$ & $2(8)$ & 0.128 & 0 & 0 & - \\
\hline Antenatal steroids & $7(44)$ & $14(31)$ & 0.835 & $7(70)$ & $14(56)$ & 0.704 & 0 & 0 & - \\
\hline Male sex & $8(50)$ & $26(58)$ & 0.289 & $5(50)$ & $16(64)$ & 0.445 & $3(50)$ & $10(51)$ & 1.000 \\
\hline Large for gestational age & $2(13)$ & $2(4)$ & 0.279 & $1(10)$ & 0 & 0.286 & $1(17)$ & $2(10)$ & 1.000 \\
\hline Apgar score-1 min & $7.0 \pm 1.6$ & $6.9 \pm 1.9$ & 0.900 & $6.3 \pm 1.3$ & $5.7 \pm 1.4$ & 0.226 & $8.2 \pm 1.3$ & $8.5 \pm 1.1$ & 0.540 \\
\hline Apgar score-5 min & $8.6 \pm 1.0$ & $8.5 \pm 1.4$ & 0.798 & $8.3 \pm 1.0$ & $7.6 \pm 1.2$ & 0.118 & $9.0 \pm 1.1$ & $9.5 \pm 0.8$ & 0.214 \\
\hline Meconium-stained fluid & 0 & $1(2)$ & 1.000 & 0 & 0 & - & 0 & $1(5)$ & 1.000 \\
\hline $\mathrm{PROM} \geq 18 \mathrm{hr}$ & $1(6)$ & $5(11)$ & 1.000 & $1(10)$ & $5(20)$ & 0.649 & 0 & $1(5)$ & 1.000 \\
\hline Pathologic chorioamnionitis & $2(13)$ & $2(4)$ & 0.279 & $2(20)$ & $1(4)$ & 0.190 & 0 & $1(5)$ & 1.000 \\
\hline PPV at birth & $4(25)$ & $9(20)$ & 0.800 & $4(40)$ & $8(32)$ & 0.849 & 0 & $1(5)$ & 0.577 \\
\hline $\begin{array}{l}\text { Time interval from birth to sur- } \\
\text { factant administration (hr) }\end{array}$ & $4.9 \pm 3.3$ & $7.7 \pm 11.1$ & 0.330 & $4.7 \pm 3.3$ & $5.2 \pm 10.1$ & 0.880 & $5.3 \pm 3.6$ & $10.9 \pm 11.7$ & 0.272 \\
\hline
\end{tabular}

Values are expressed as mean \pm standard deviation or number (\%). A $P<0.05$ represents statistically significant. Abbreviations: GA, gestational age; MIST, minimally invasive surfactant therapy; DM, diabetes mellitus; HTN, hypertension; PROM, premature rupture of membrane; PPV, positive pressure ventilation. 
Table 2. Respiratory Outcomes and Relevant Morbidities

\begin{tabular}{|c|c|c|c|c|c|c|c|c|c|}
\hline \multirow[b]{2}{*}{ Variable } & \multicolumn{3}{|c|}{ Total $(n=61)$} & \multicolumn{3}{|c|}{$\mathrm{GA}_{30} 0^{+0}-34^{+6}(\mathrm{n}=35)$} & \multicolumn{3}{|c|}{ GA $35^{+0}-41^{+6}(n=26)$} \\
\hline & $\begin{array}{c}\text { MIST } \\
(n=16)\end{array}$ & $\begin{array}{c}\text { Control } \\
(n=45)\end{array}$ & $\begin{array}{c}P \text { - } \\
\text { value }\end{array}$ & $\begin{array}{l}\text { MIST } \\
(n=10)\end{array}$ & $\begin{array}{c}\text { Control } \\
(n=25)\end{array}$ & $\begin{array}{c}P \text { - } \\
\text { value }\end{array}$ & $\begin{array}{l}\text { MIST } \\
(n=6)\end{array}$ & $\begin{array}{l}\text { Control } \\
(n=20)\end{array}$ & $\begin{array}{c}P \text { - } \\
\text { value }\end{array}$ \\
\hline Failure of procedure on the 1st attempt & $1(6)$ & $9(20)$ & 0.267 & $1(10)$ & $4(16)$ & 1.000 & 0 & $5(25)$ & 0.298 \\
\hline MV anytime & $1(6)$ & $37(82)$ & $<0.001$ & 0 & $20(80)$ & $<0.001$ & $1(17)$ & $17(85)$ & 0.004 \\
\hline Need for $>1$ dose of surfactant & $6(38)$ & $4(9)$ & 0.015 & $4(40)$ & $2(8)$ & 0.043 & $2(33)$ & $2(10)$ & 0.218 \\
\hline Bradycardia & $2(13)$ & $20(44)$ & 0.033 & $1(10)$ & $9(36)$ & 0.218 & $1(17)$ & $11(55)$ & 0.170 \\
\hline Air-leak syndrome & $4(25)$ & $8(18)$ & 0.715 & $2(20)$ & $2(8)$ & 0.561 & $2(33)$ & $6(30)$ & 1.000 \\
\hline Pulmonary hemorrhage & 0 & $2(4)$ & 1.000 & 0 & $1(4)$ & 1.000 & 0 & $1(5)$ & 1.000 \\
\hline BPD & 0 & 0 & - & 0 & 0 & - & 0 & 0 & - \\
\hline Duration of MV (d) & $0.3 \pm 1.0$ & $3.6 \pm 2.7$ & 0.000 & $0.0 \pm 0.0$ & $2.7 \pm 2.2$ & 0.000 & $0.7 \pm 1.6$ & $4.9 \pm 2.9$ & 0.003 \\
\hline Duration of MV+CPAP (d) & $3.5 \pm 1.7$ & $4.7 \pm 3.1$ & 0.134 & $3.6 \pm 1.4$ & $3.6 \pm 2.6$ & 1.000 & $3.3 \pm 2.3$ & $6.2 \pm 3.2$ & 0.054 \\
\hline Duration of MV+CPAP+HHHFNC (d) & $7.4 \pm 4.1$ & $7.6 \pm 3.9$ & 0.846 & $8.3 \pm 4.9$ & $7.1 \pm 4.1$ & 0.472 & $5.8 \pm 1.9$ & $8.2 \pm 3.6$ & 0.143 \\
\hline Hospital stay (d) & $23.6 \pm 14.8$ & $29.7 \pm 17.2$ & 0.217 & $30.5 \pm 14.7$ & $39.6 \pm 16.8$ & 0.144 & $12.2 \pm 4.1$ & $17.3 \pm 5.9$ & 0.035 \\
\hline PDA requiring treatment & $10(63)$ & $24(53)$ & 0.781 & $6(60)$ & $15(60)$ & 1.000 & $4(67)$ & $9(45)$ & 0.645 \\
\hline NEC $\geq$ stage 2 & 0 & 0 & - & 0 & 0 & - & 0 & 0 & - \\
\hline ROP & 0 & 0 & - & 0 & 0 & - & 0 & 0 & - \\
\hline IVH $\geq$ stage 2 & 0 & $1(2)$ & 1.000 & 0 & $1(4)$ & 1.000 & 0 & 0 & - \\
\hline HIE & $1(6)$ & $10(22)$ & 0.259 & $1(10)$ & $4(16)$ & 1.000 & 0 & $6(30)$ & 0.280 \\
\hline Suspected sepsis & $12(75)$ & $41(91)$ & 0.189 & $6(60)$ & $23(92)$ & 0.043 & $6(100)$ & $18(90)$ & 1.000 \\
\hline Culture proven sepsis & 0 & 0 & - & 0 & 0 & - & 0 & 0 & - \\
\hline
\end{tabular}

Values are expressed as number (\%) or mean \pm standard deviation. A $P<0.05$ represents statistically significant.

Abbreviations: GA, gestational age; MIST, minimally invasive surfactant therapy; MV, mechanical ventilation; BPD, bronchopulmonary dysplasia; CPAP, continuous positive airway pressure; HHHFNC, heated humidified high flow nasal cannula; PDA, patent ductus arteriosus; NEC, necrotizing enterocolitis; ROP, retinopathy of prematurity; IVH, intraventricular hemorrhage; HIE, hypoxic ischemic encephalopathy.

control group was, in all study populations $(P<0.001)$ (Table 2$)$. Compared to neonates in the control group, those in the MIST group more frequently required additional administration of pulmonary surfactant $(P=0.015)$ (Table 2 , Figure $2 A)$. The incience of bradycardia during pulmonary surfactant administration was significantly lower in the MIST group than in the control group ( $P=0.033$ ) (Table 2 , Figure $2 \mathrm{~B}$ ). In contrast, there were no significant differences in the occurrence of air-leak syndrome between the groups (Table 2). The duration of mechanical ventilation was significantly shorter in the MIST group than in the control group (Table 2, Figure 2C). For the subgroup with a gestational age of 35 weeks or more, the duration of hospital stay was significantly shorter in the MIST group than in the control group $(P=0.035)$ (Table 2, Figure 2D).

\section{Multiple logistic regression}

To analyze independent factors, multiple logistic regression analysis was performed for each gestational age group for variables with $P<0.05$ in Tables 1, 2 (Table 3). Altogether, the
MIST group was significantly less likely to receive mechanical ventilation during the hospitalization period than the control group was, independently $(P<0.001$; risk ratio [RR], 0.014; 95\% confidence interval [CI], 0.002 to 0.125 ). The number of patients requiring more than secondary administration of pulmonary surfactant was significantly higher in the MIST group than in the control group ( $P=0.008 ; \mathrm{RR}, 6.150 ; 95 \% \mathrm{CI}, 1.455$ to 26.001 ). Further, the incidence of bradycardia at the time of pulmonary SRT was significantly lower in the MIST group than in the control group ( $P=0.022 ; \mathrm{RR}, 0.179 ; 95 \% \mathrm{CI}, 0.036$ to 0.879 ). During the clinical course, the duration of mechanical ventilation in the MIST group was found to have a statistically significant effect independently; however, there was no significant effect on the hospitalization period.

\section{DISCUSSION}

Surfactant application by MIST with tracheal catheterization 

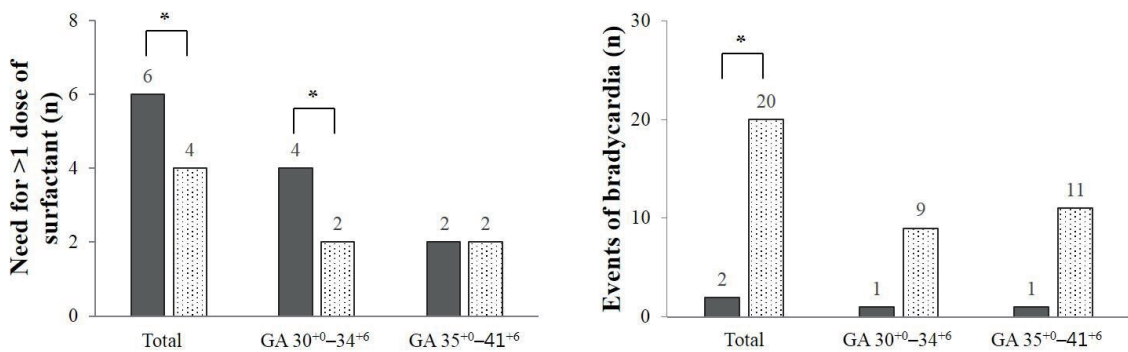

A

$\square$ MIST $₫$ Control

B
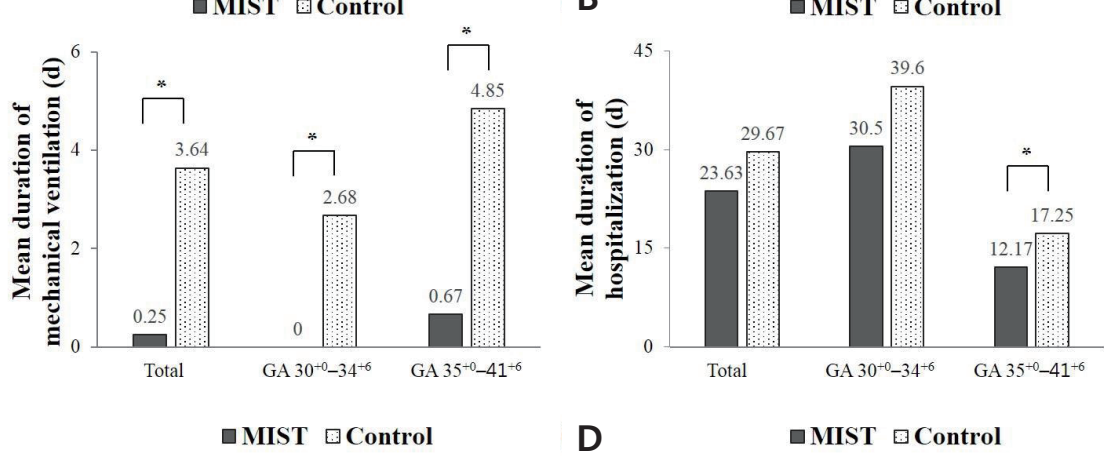

Figure 2. Neonatal clinical outcomes: (A) need for $>1$ dose of surfactant, (B) events for bradycardia, (C) mean duration of mechanical ventilation, (D) mean duration of hospitalization. Values are expressed as mean. *Statistically significant $(P<0.05)$. Abbreviations: GA, gestational age; MIST, minimally invasive surfactant therapy.

Table 3. Effect Estimate (RR) of MIST Group Over Non-MIST Group

\begin{tabular}{|c|c|c|c|c|c|c|c|c|c|}
\hline \multirow[b]{2}{*}{ Variable } & \multicolumn{3}{|c|}{ Total $(n=61)$} & \multicolumn{3}{|c|}{ GA $30^{+0}-34^{+6}(n=35)$} & \multicolumn{3}{|c|}{ GA $35^{+0}-41^{+6}(n=26)$} \\
\hline & $\begin{array}{c}\text { Effect } \\
\text { estimate } \\
\text { (RR) }\end{array}$ & $95 \% \mathrm{CI}$ & $\begin{array}{c}P \text { - } \\
\text { value }\end{array}$ & $\begin{array}{c}\text { Effect } \\
\text { estimate } \\
\text { (RR) }\end{array}$ & $95 \% \mathrm{CI}$ & $\begin{array}{c}P \text { - } \\
\text { value }\end{array}$ & $\begin{array}{c}\text { Effect } \\
\text { estimate } \\
\text { (RR) }\end{array}$ & $95 \% \mathrm{CI}$ & $\begin{array}{c}P \text { - } \\
\text { value }\end{array}$ \\
\hline MV anytime & $\mathrm{RR}, 0.014$ & 0.002 to 0.125 & 0.000 & $\mathrm{RR}, 0.200$ & 0.091 to 0.438 & 0.000 & $\mathrm{RR}, 0.035$ & 0.003 to 0.419 & 0.001 \\
\hline Need for $>1$ dose of surfactant & $\mathrm{RR}, 6.150$ & 1.455 to 26.001 & 0.008 & $\mathrm{RR}, 7.667$ & 1.123 to 52.321 & 0.023 & $\mathrm{RR}, 4.500$ & 0.479 to 42.248 & 0.165 \\
\hline Bradycardia & RR, 0.179 & 0.036 to 0.879 & 0.022 & RR, 0.198 & 0.021 to 1.822 & 0.124 & $\mathrm{RR}, 0.164$ & 0.016 to 1.666 & 0.099 \\
\hline Duration of MV (d) & MD, 3.394 & 2.436 to 4.353 & 0.000 & $\mathrm{MD}, 2.680$ & 1.759 to 3.601 & 0.001 & MD, 4.183 & 1.612 to 6.754 & 0.004 \\
\hline Hospital stay (d) & MD, 6.042 & -3.636 to 15.719 & 0.210 & MD, 9.100 & -3.282 to 21.482 & 0.136 & MD, 5.083 & -0.282 to 10.449 & 0.059 \\
\hline $\begin{array}{l}\text { Antibiotics therapy for suspected } \\
\text { infection }\end{array}$ & $\mathrm{RR}, 0.293$ & 0.064 to 1.349 & 0.101 & $\mathrm{RR}, 0.130$ & 0.019 to 0.890 & 0.023 & $\mathrm{RR}, 0.900$ & 0.778 to 1.042 & 0.420 \\
\hline
\end{tabular}

A $P<0.05$ represents statistically significant.

Abbreviations: RR, relative risk ratio; MIST, minimally invasive surfactant therapy; GA, gestational age; CI, confidence interval; MV, mechanical ventilation; $\mathrm{MD}$, mean difference.

is now recognized as one of the standard methods of SRT in preterm infants with a gestational age less than 30 weeks who are diagnosed with RDS, and it may improve their short-term and long-term outcomes. However, studies concerning the use of MIST in neonates with gestational ages of 30 weeks or more are insufficient, and the results are inconclusive ${ }^{17)}$. Our study showed that MIST can safely be used and that it improves the short-term clinical outcomes in newborns with a gestational age of 30 weeks or more who are diagnosed with RDS.

Early noninvasive forms of ventilation such as CPAP have been applied as a primary strategy for the management of RDS in very-low-birth-weight infants with spontaneous breathing ${ }^{18)}$. This is because the risk of airway and lung injury and respiratory tract infection, which is commonly associated with invasive mechanical ventilation, is relatively low in noninvasive ventilation ${ }^{19)}$. However, if neonates who received only noninvasive 
ventilation without SRT at the time of birth show a clear sign of RDS later, the surfactant should be eventually administered through endotracheal intubation. To overcome this problem, Verder et al. ${ }^{4)}$ devised the INSURE method. However, this method also required endotracheal intubation; therefore, some physical concerns such as tracheal or laryngeal injury remained ${ }^{20)}$. For this reason, some researchers modified the INSURE method to devise a new method, namely MIST ${ }^{21)}$. The MIST procedure is commonly referred to as the less invasive surfactant administration (LISA). Thus, the European Consensus Guidelines, newly published in $2016^{2)}$ added that INSURE should be considered in newborns with CPAP failure and that MIST can be substituted for INSURE in spontaneously breathing infants.

As observed in previous studies ${ }^{10,11)}$, the rate of application of mechanical ventilation was significantly reduced by the LISA method in this study; that is, it was lower in the MIST group than in the control group. Tomar et al. ${ }^{22)}$ published a study using a modified MIST method (Magill forceps are not used in this method, and the gastric tube is directly inserted into the airway by the practitioner). According to this study, in newborn infants with a gestational age of $24^{+0}$ to $33^{+6}$ weeks who were diagnosed with RDS, the durations of mechanical ventilation and CPAP support were significantly lower in the modified MIST group than in the INSURE group; however, there were no differences in the need for mechanical ventilation within the first 72 hours, and in the other neonatal morbidities and mortalities, between the groups.

In this study, the incidence of bradycardia during surfactant administration was significantly lower in the MIST group than in the control group in multiple regression analysis. In most cases, bradycardia results from vagal nerve reflex caused by uncomfortable stimulation of the pharynx and larynx during neonatal endotracheal intubation. This bradycardia is usually referred to as "stable bradycardia," because it can be easily nor malized by temporarily suspending intubation or by sufficient oxygenation $^{23)}$. MIST does not require endotracheal intubation; hence, it can be inferred that the occurrence of bradycardia, which is an adverse effect that can occur during endotracheal intubation, is reduced. Meanwhile, the American Academy of Pediatrics $^{24)}$ recommends the use of premedication for endotracheal intubation in neonates, except in emergency situations. This premedication includes analgesics, sedatives, and muscle relaxants. Further, vagolytic agents can be used to prevent bradycardia that can occur during endotracheal intubation. However, according to a European survey published in 2017, 52\% of neonatologists do not use premedication when administering MIST $^{25)}$. This is because spontaneous breathing plays a major role in the proper distribution of the pulmonary surfactant in the lungs in MIST, and the premedication can reduce the respiratory effort in premature infants in particular ${ }^{26)}$.

Meanwhile, Dargaville et al. ${ }^{27)}$ showed that the incidence of pneumothorax reduced from $8.0 \%$ to $2.4 \%$ after introducing MIST with a vascular catheter in neonates aged 29 to 32 weeks who were diagnosed with RDS. However, in our study, there was no significant difference in the occurrence of air-leak syndrome including pneumothorax between the two groups of neonates.

According to a recent analysis released in 2017 concerning the results of LISA treatment in premature infants, there was no significant difference between the LISA group and the con trol group with regard to the need for additional surfactant administration after primary pulmonary SRT ${ }^{17)}$. Meanwhile, animal studies published in 2005 showed that the distribution of pulmonary surfactant was better in the noninvasive respiratory assist group than in the mechanical ventilation group, in spontaneously breathing rabbits ${ }^{19)}$. However, in our study, compared to the control group, the need for additional administration of pulmonary surfactant was increased in the MIST group. These results may be attributable to the lack of experience of the operator with regard to MIST or the anxiety caused by it. Additional studies are warranted to explain the results that are different from those of the previous studies.

We subdivided each group into two more groups according to gestational age, with neonates with gestational age between 30 and 35 weeks in one group and those with age $\geq 35$ weeks in the other group. The reasons for dividing the neonates into two groups based on the gestational age of 35 weeks as the cut off are as follows. Moderately premature infants, i.e., newborns with a gestational age of more than 30 weeks and less than 35 weeks, are known to contribute to increased neonatal morbidity. Although extremely preterm births are well known to have a higher risk of poor outcome, in practice, the number of moderately premature infant births is relatively higher, which may be perceived as a major health problem. According to a Swedish national population-based study published in $2011^{11)}$, transient tachypnea of the newborn or RDS was observed in about one-third of moderate premature infants. Therefore, subgroup analysis was performed to determine whether morbidity was higher in moderately premature infants. 
The limitations of this study may be that it was retrospective in nature, the number of subjects was small, randomization among the comparison groups was not possible, and the clinical severity of the two groups might have differed from the beginning. Another limitation was that the control group could not be subdivided into the INSURE group and the mechanical ventilation maintenance group, because the sample size of the INSURE group was too small. This was attributed to the cutbacks in the insurance amount for INSURE during the study period, and to the fact that INSURE was not performed as a routine procedure and was executed with early extubation after invasive ventilator application. Therefore, additional studies to address these limitations are warranted.

In conclusion, MIST is considered a relatively safe and effective procedure for treating RDS, and for reducing the need for mechanical ventilation and the occurrence of endotracheal intubation-induced bradycardia. For well-experienced practitioners, MIST may be an appropriate choice of treatment for RDS in neonates with a gestational age of 30 weeks or more.

\section{CONFLICT OF INTEREST}

No potential conflict of interest relevant to this article was reported.

\section{REFERENCES}

1. Speer CP. Neonatal respiratory distress syndrome: an inflam matory disease? Neonatology 2011;99:316-9.

2. Sweet DG, Carnielli V, Greisen G, Hallman M, Ozek E, Plavka $\mathrm{R}$, et al. European consensus guidelines on the management of respiratory distress syndrome: 2016 update. Neonatology 2017;111:107-25.

3. Committee on Fetus and Newborn; American Academy of Pediatrics. Respiratory support in preterm infants at birth. Pediatrics 2014;133:171-4.

4. Verder H, Robertson B, Greisen G, Ebbesen F, Albertsen P, Lundstrom K, et al. Surfactant therapy and nasal continuous positive airway pressure for newborns with respiratory distress syndrome. Danish-Swedish Multicenter Study Group. N Engl J Med 1994;331:1051-5.

5. Verder H, Albertsen P, Ebbesen F, Greisen G, Robertson B, Bertelsen A, et al. Nasal continuous positive airway pressure and early surfactant therapy for respiratory distress syndrome in newborns of less than 30 weeks' gestation. Pediatrics 1999; 103:E24.

6. Venkatesh V, Ponnusamy V, Anandaraj J, Chaudhary R, Malviya M, Clarke P, et al. Endotracheal intubation in a neonatal pop ulation remains associated with a high risk of adverse events. Eur J Pediatr 2011;170:223-7.

7. Nilsson R, Grossmann G, Robertson B. Bronchiolar epithelial lesions induced in the premature rabbit neonate by short pe riods of artificial ventilation. Acta Pathol Microbiol Scand A 1980;88:359-67.

8. Kolatat T, Aunganon $\mathrm{K}$, Yosthiem P. Airway complications in neonates who received mechanical ventilation. J Med Assoc Thai 2002;85 Suppl 2:S455-62.

9. Allen KA. Premedication for neonatal intubation: which medications are recommended and why. Adv Neonatal Care 2012; 12:107-11.

10. Shim GH. Update of minimally invasive surfactant therapy. Korean J Pediatr 2017;60:273-81.

11. Gopel W, Kribs A, Ziegler A, Laux R, Hoehn T, Wieg C, et al. Avoidance of mechanical ventilation by surfactant treatment of spontaneously breathing preterm infants (AMV): an openlabel, randomised, controlled trial. Lancet 2011;378:1627-34.

12. Gortner L, Bartmann P, Pohlandt F, Bernsau U, Porz F, Hellwege $\mathrm{HH}$, et al. Early treatment of respiratory distress syndrome with bovine surfactant in very preterm infants: a multicenter controlled clinical trial. Pediatr Pulmonol 1992;14:4-9.

13. Jobe AH, Bancalari E. Bronchopulmonary dysplasia. Am J Respir Crit Care Med 2001;163:1723-9.

14. Kliegman RM, Walsh MC. Neonatal necrotizing enterocolitis: pathogenesis, classification, and spectrum of illness. Curr Probl Pediatr 1987;17:213-88.

15. International Committee for the Classification of Retinopathy of Prematurity. The International Classification of Retinopathy of Prematurity revisited. Arch Ophthalmol 2005;123:991-9.

16. Papile LA, Burstein J, Burstein R, Koffler H. Incidence and evolution of subependymal and intraventricular hemorrhage: a study of infants with birth weights less than 1,500 gm. J Pediatr 1978;92:529-34.

17. Aldana-Aguirre JC, Pinto M, Featherstone RM, Kumar M. Less invasive surfactant administration versus intubation for surfactant delivery in preterm infants with respiratory distress syndrome: a systematic review and meta-analysis. Arch Dis Child Fetal Neonatal Ed 2017;102:F17-23.

18. Lopez E, Gascoin G, Flamant C, Merhi M, Tourneux P, Baud O, et al. Exogenous surfactant therapy in 2013: what is next? Who, when and how should we treat newborn infants in the future? BMC Pediatr 2013;13:165.

19. Bohlin K, Bouhafs RK, Jarstrand C, Curstedt T, Blennow M, 
Robertson B. Spontaneous breathing or mechanical ventilation alters lung compliance and tissue association of exogenous surfactant in preterm newborn rabbits. Pediatr Res 2005;57:62430.

20. Klebermass-Schrehof K, Wald M, Schwindt J, Grill A, Prusa AR, Haiden N, et al. Less invasive surfactant administration in extremely preterm infants: impact on mortality and morbidity. Neonatology 2013;103:252-8.

21. Aguar M, Cernada M, Brugada M, Gimeno A, Gutierrez A, Vento M. Minimally invasive surfactant therapy with a gastric tube is as effective as the intubation, surfactant, and extubation technique in preterm babies. Acta Paediatr 2014;103:e229-33.

22. Tomar RS, Ghuliani R, Yadav D. Effect of surfactant therapy using orogastric tube for tracheal catheterization in preterm newborns with respiratory distress. Indian J Pediatr 2017;84: 257-61.
23. Jones P, Dauger S, Peters MJ. Bradycardia during critical care intubation: mechanisms, significance and atropine. Arch Dis Child 2012;97:139-44.

24. Kumar P, Denson SE, Mancuso TJ; Committee on Fetus and Newborn, Section on Anesthesiology and Pain Medicine. Premedication for nonemergency endotracheal intubation in the neonate. Pediatrics 2010;125:608-15.

25. Klotz D, Porcaro U, Fleck T, Fuchs H. European perspective on less invasive surfactant administration-a survey. Eur J Pediatr 2017;176:147-54.

26. Kanmaz HG, Erdeve O, Canpolat FE, Mutlu B, Dilmen U. Surfactant administration via thin catheter during spontaneous breathing: randomized controlled trial. Pediatrics 2013;131: e502-9.

27. Dargaville PA, Ali SKM, Jackson HD, Williams C, De Paoli AG. Impact of minimally invasive surfactant therapy in preterm infants at 29-32 weeks gestation. Neonatology 2018;113:7-14. 